\title{
INDECOMPOSABLE REPRESENTATIONS AND THE LOOP-SPACE OPERATION ${ }^{1}$
}

ALEX HELLER

For a certain class of algebras, including group-algebras of finite groups, we shall introduce a permutation in the set of isomorphismclasses of nonprojective indecomposable modules. This permutation is in essence given by the loop-space functor [2]. It is to be hoped that the study of this permutation may give some insight into the difficult problem of classifying indecomposable representations. In any case we append here some examples, showing that the orbits may be either finite or infinite, and that even in simple cases there may be infinitely many of them.

1. The loop-space operation. We denote by $\Lambda$ a finite dimensional algebra with unit 1 over a field $K$. By "module" we shall always mean a finitely generated left $\Lambda$-module on which 1 operates as the identity. We shall say that $\Lambda$ is weak-Frobenius if the classes of injective and projective $\Lambda$-modules coincide. Quasi-Frobenius algebras, Frobenius algebras, symmetric algebras and in particular group algebras of finite groups are all weak-Frobenius.

For any module $A$ we denote by $[A]$ the isomorphism class of $A$. If $B$ is also a module we set $[A]+[B]=[A \oplus B]$. We say that $[A]$ is indecomposable when $A$ is; the Krull-Schmidt theorem asserts that for any $A$,

$$
[A]=\sum\left[A_{i}\right]
$$

with the $\left[A_{i}\right]$ indecomposables determined by $[A]$.

It follows in particular that $A \approx A^{0} \oplus A^{p}$ where $A^{p}$ is projective and $A^{0}$ contains no projective direct summand, and that $\left[A^{0}\right]$ and $\left[A^{p}\right]$ depend only on $[A]$. We shall write $\left[A^{0}\right]=[A]^{0}$.

The loop-space functor is defined by the following process. For each module $A$, map a projective by an epimorphism onto $A$; the kernel is the loop-space of $A$, denoted by $\Omega A$. But if $0 \rightarrow B \rightarrow X \rightarrow A \rightarrow 0$ is exact and $X$ is projective then $B^{0}$ depends, up to isomorphism, only on $[A]$. For if $0 \rightarrow B^{\prime} \rightarrow X^{\prime} \rightarrow A \rightarrow 0$ also has these properties then $B \oplus X^{\prime} \approx B^{\prime} \oplus X$ (Schanuel, cf. [4]). We write $\Omega[A]=\left[B^{0}\right]$.

Since the direct sum of two exact sequences is exact, and the direct

Received by the editors August 31, 1960.

1 This work was in part supported by National Science Foundation Grant NSF-G12912. 
s.ım of two projectives is projective we have for any two modules $A$ and $B$

$$
\Omega([A]+[B])=\Omega[A]+\Omega[B] .
$$

We have also, of course, $\Omega[X]=[0]$ for $X$ projective, and thus $\Omega\left[A^{0}\right]=\Omega[A]$.

Dually we may write $A=A^{0 *} \oplus A^{i}$ where $A^{i}$ is injective and $A^{0 *}$ has no injective summands, and define $\Omega^{*}[A]=\left[B^{0 *}\right]$ where $0 \rightarrow A$ $\rightarrow Y \rightarrow B \rightarrow 0$ is exact and $Y$ is injective.

If $\Lambda$ has the property that the classes of injective and projective modules coincide, and $A$ is a module, then clearly

$$
\Omega^{*} \Omega[A]=\Omega \Omega^{*}[A]=[A]^{0} .
$$

Thus $\Omega$ and $\Omega^{*}$ are inverse permutations of the set of isomorphism classes of modules having no projective direct summands. But then (1.1) implies immediately the following result.

Proposition 1. If $\Lambda$ is a weak-Frobenius algebra then $\Omega$ and $\Omega^{*}$ are inverse permutations of the set of isomorphism classes of nonprojective indecomposable $\Lambda$-modules.

We denote by $M(\Lambda)$ the set of isomorphism classes of indecomposable $\Lambda$-modules, and by $M^{0}(\Lambda)$ the subset of nonprojective ones, so that $M^{p}(\Lambda)=M(\Lambda)-M^{0}(\Lambda)$ consists of the projective indecomposables. It follows quickly from the Krull-Schmidt theorem that $M^{p}(\Lambda)=\left\{\left[\Lambda e_{1}\right], \cdots,\left[\Lambda e_{n}\right]\right\}$ where $\left\{e_{1}, \cdots, e_{n}\right\}$ is a maximal set of orthogonal idempotents [5]. Thus $M(\Lambda)$ is finite if and only if $M^{0}(\Lambda)$ is.

Proposition 2. If $\Lambda$ is a weak-Frobenius algebra then $M(\Lambda)$ is finite if and only if (a) each $\Omega$-orbit in $M^{0}(\Lambda)$ is finite, (b) the number of $\Omega$-orbits in $M^{0}(\Lambda)$ is finite.

We conclude these generalities by examining the effect of a "change of algebras." Suppose that $\Gamma$ is also a $K$-algebra, and that $\phi: \Gamma \rightarrow \Lambda$ is an algebra homomorphism. If $A$ is a $\Lambda$-module we denote by $\phi^{*}[A]$ the class of $A$ converted into a $\Gamma$-module by the use of $\phi$. We have clearly

$$
\phi^{*}([A]+[B])=\phi^{\sharp}[A]+\phi^{*}[B] .
$$

However, $\phi^{\sharp}[A]$ need not be indecomposable even when $[A]$ is.

If further $\Lambda$ is projective as a $\Gamma$-module we have for any $\Lambda$-module $A$

$$
\left(\phi^{*}\left[A^{0}\right]\right)^{0}=\left(\phi^{*}[A]\right)^{0}
$$


and thus

$$
\Omega \phi^{\#}=\phi^{\#} \Omega,
$$

this resulting directly from the definition of $\Omega$.

2. Examples. We consider three cases. In each one the algebra is in fact symmetric, and thus a fortiori weak-Frobenius.

For the first, let $x$ be an indeterminate over $K$ and set $\Lambda=K[x] /\left(x^{n}\right)$ where $n>1$. Then $M^{p}(\Lambda)=\{[\Lambda]\}$ and $M^{0}(\Lambda)=\left\{\left[V_{1}\right], \cdots,\left[V_{n-1}\right]\right\}$ where $V_{q}=K[x] /\left(x^{q}\right)$ is regarded as a quotient module of $\Lambda .^{2}$ We have clearly $\Omega\left[V_{q}\right]=\left[V_{n-q}\right]$.

Next, let $x$ and $y$ be indeterminates over $K$ and set

$$
\Lambda=K[x, y] /\left(x^{n}, y^{n}\right)
$$

where $n>1$. Then, once more, $M^{p}(\Lambda)=\{[\Lambda]\}$. No complete description of $M^{0}(\Lambda)$ has been given and indeed it is indicated in [3] that it may well be difficult to find one. We have however the following result.

ThEOREM. If $\Lambda=K[x, y] /\left(x^{n}, y^{n}\right)$ then $M^{0}(\Lambda)$ contains both finite and infinite $\Omega$-orbits; moreover if $n>2$ there are infinitely many infinite orbits.

First, suppose $A=K[x, y] /\left(x^{q}, y^{n}\right)$, where $q<n$, regarded as a quotient of $\Lambda$. We may also write

$$
\Lambda=K[x] /\left(x^{n}\right) \otimes K[y] /\left(y^{n}\right), \quad A=K[x] /\left(x^{q}\right) \otimes K[y] /\left(y^{n}\right) .
$$

Comparing with the first example, we see that $\Omega^{2}[A]=[A]$.

In order to exhibit infinite orbits we let $X$ and $Y$ be projective resolutions of $K$ over $\Lambda^{\prime}=K[x] /\left(x^{n}\right)$ and $\Lambda^{\prime \prime}=K[y] /\left(y^{n}\right)$. Then $X \otimes_{K} Y$ is a projective resolution of $K$ over $\Lambda=\Lambda^{\prime} \otimes_{K} \Lambda^{\prime \prime}$. But $\left(X \otimes_{K} Y\right) \otimes_{\Lambda} K=\left(X \otimes_{\Lambda^{\prime}} K\right) \otimes_{K}\left(Y \otimes_{\Lambda^{\prime}}, K\right)$ and thus, by the Kunneth theorem, $\operatorname{Tor}_{n}^{\Lambda}(K, K) \approx K^{n+1}$.

Next we consider the modules defined by

$$
\begin{aligned}
& W_{q}=K a_{1} \oplus \cdots \oplus K a_{q} \oplus K b_{0} \oplus \cdots \oplus K b_{q}, \\
& x a_{i}=b_{i-1}, \quad y a_{i}=b_{i}, \quad x b_{i}=y b_{i}=0 .
\end{aligned}
$$

These are all indecomposable (cf. [3, Proposition 5]). Each occurs in an exact sequence $0 \rightarrow K^{q+1} \rightarrow W_{q} \rightarrow K^{q} \rightarrow 0$. Comparing dimensions in the exact sequence of Tor's we see that $\operatorname{dim} \operatorname{Tor}_{n}^{\Lambda}\left(W_{q}, K\right) \geqq n+1-q$.

On the other hand it follows immediately from the definition of $\Omega$ that $\operatorname{Tor}_{n}^{\Lambda}(\Omega A, B) \approx \operatorname{Tor}_{n+1}^{\Lambda}(A, B)$ for $n \geqq 1$. In particular if the orbit of

2 This is a well-known theorem on nilpotent matrices. 
$[A]$ is finite then $\operatorname{Tor}_{n}^{\Lambda}(A, B)$ is periodic in $n$ for $n \geqq 1$. This is clearly not the case for $A=W_{q}$; we conclude that each $\left[W_{q}\right]$ belongs to an infinite orbit.

It remains only to show that when $n>2$ the $\left[W_{q}\right.$ ] belong to distinct orbits. To see this write $\phi: K[x] /\left(x^{n}\right) \rightarrow \Lambda$ for the "inclusion" homomorphism. We have clearly

$$
\phi^{\sharp}\left[W_{q}\right]=q\left[V_{2}\right]+\left[V_{1}\right]
$$

where the $\left[V_{i}\right]$ are as in the first example. Moreover $\Lambda$ is $K[x] /\left(x^{n}\right)$ free and thus by 1.3 ,

$$
\phi^{*} \Omega^{k}\left[W_{q}\right]=\left\{\begin{array}{lr}
q\left[V_{2}\right]+\left[V_{1}\right], & k \text { even, } \\
q\left[V_{n-2}\right]+\left[V_{n-1}\right], & k \text { odd, }
\end{array}\right.
$$

which implies the result.

For the last example let $\pi$ be a generalized quaternion group and suppose that $K$ has characteristic 2 . If $\Lambda=K \pi$ then $M^{p}(\Lambda)=\{[\Lambda]\}$. But since $Z_{2} \oplus Z_{2}$ is a quotient group of $\pi$ we have $M\left(K[x, y] /\left(x^{2}, y^{2}\right)\right)$ $C M(\Lambda)$, and $M^{0}(\Lambda)$ is consequently infinite. On the other hand there is [1, Chapter XII, p. 7] a projective resolution of $K$ over $\Lambda$ which is periodic of period 4 and thus $\Omega^{4}$ is the identity on $M^{0}(\Lambda)$. Since each orbit is finite the class of orbits must of course be infinite.

\section{BIBLIOGRAPHY}

1. H. Cartan and S. Eilenberg, Homological algebra, Princeton, 1957.

2. A. Heller, The loop-space functor in homological algebra, Trans. Amer. Math. Soc. vol. 96 (1960) pp. 382-394.

3. A. Heller and I. Reiner, Indecomposable representations, Illinois J. Math. vol. 5 (1961) pp. 314-323.

4. I. Kaplansky, Homological dimension of rings and modules, University of Chicago, 1959 (mimeographed notes).

5. H. Nagao and T. Nakayama, On the structure of $\left(M_{0}\right)$ - and $\left(M_{u}\right)$-modules, Math. Z. vol. 59 (1953) pp. 164-170.

UNIVERSITY OF ILLINOIS 